\title{
LES COLLÉGIENS ET LA TRANSMISSION FAMILIALE D'UN CAPITAL INFORMATIQUE
}

Cédric Fluckiger

Presses de Sciences Po (P.F.N.S.P.) | «Agora débats/jeunesses »

2007/4 N 46 | pages 32 à 42

ISSN 1268-5666

ISBN 9782296050419

Article disponible en ligne à l'adresse :

http://www.cairn.info/revue-agora-debats-jeunesses-2007-4-page-32.htm

\section{Pour citer cet article :}

Cédric Fluckiger, « Les collégiens et la transmission familiale d'un capital informatique », Agora débats/jeunesses 2007/4 (N46), p. 32-42.

DOI 10.3917/agora.046.0032

Distribution électronique Cairn.info pour Presses de Sciences Po (P.F.N.S.P.).

(C) Presses de Sciences Po (P.F.N.S.P.). Tous droits réservés pour tous pays.

La reproduction ou représentation de cet article, notamment par photocopie, n'est autorisée que dans les limites des conditions générales d'utilisation du site ou, le cas échéant, des conditions générales de la licence souscrite par votre établissement. Toute autre reproduction ou représentation, en tout ou partie, sous quelque forme et de quelque manière que ce soit, est interdite sauf accord préalable et écrit de l'éditeur, en dehors des cas prévus par la législation en vigueur en France. Il est précisé que son stockage dans une base de données est également interdit. 


\section{LES COLLÉGIENS ET LA TRANSMISSION FAMILIALE D'UN CAPITAL INFORMAATIQUE}

Bien que ce soit avant tout dans le groupe de pairs que les adolescents trouvent des ressources pour s'approprier les outils informatiques, leurs usages des ordinateurs se déroulent en partie au sein de la famille. Cet article vise à étudier les modalités concrètes d'appropriation des technologies informatiques dans le cadre familial, en introduisant et en questionnant la notion de " capital informatique ".

\section{Cédric Fluckiger}

Doctorant en sciences de l'éducation (thèse sur le processus d'appropriation des outils informatiques par les jeunes adolescent) à l'École normale supérieure de Cachan au sein du laboratoire Sciences techniques enseignements et formation, ainsi qu'au Laboratoire de sociologie des usages et économie (SENSE - Sociology and Economics of Networks and Services) de France Télécom Recherche et développement Cédric Fluckiger FTRD/TECH/SENSE France Télécom R\&D 38-40, avenue du Général Leclerc 92130 Issy-les-Moulineaux Courriel : cedric.fluckiger@orange-ftgroup.com 
De par son omniprésence dans les pratiques culturelles, médiatiques et de communication des adolescents, l'ordinateur a l'air d'être une " affaire de jeunes ". Les adolescents se distinguent par de forts taux d'équipement et de

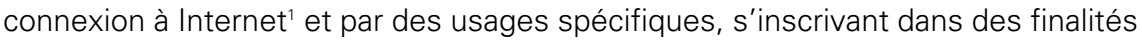
surtout ludiques, essentiellement autour de $\mathrm{MSN}^{2}$, des blogs et des jeux vidéo. Mais l'ordinateur n'est pas seulement un objet technique que I'on doit s'approprier, il s'insère également dans des stratégies de reconnaissance identitaire et d'autonomisation ${ }^{3}$. Ces usages juvéniles dessinent les contours d'une culture informatique spécifique aux jeunes générations et deviennent un instrument pour la manifestation publique du statut d'adolescent, qui nécessite une constante réaffirmation ${ }^{4}$.

Ces usages requièrent la maîtrise d'un ensemble varié de compétences techniques (relatives à la manipulation instrumentale) et relationnelles (comme les normes et les codes de la communication écrite synchrone). C'est avant tout dans le groupe de pairs que les adolescents trouvent les ressources pour l'appropriation des outils informatiques, qu'ils prennent connaissance des sites ou des blogs à fréquenter ou apprennent l'existence des logiciels de téléchargement ou de messagerie instantanée. Or, la prédominance de la socialisation horizontale dans le processus d'appropriation risque de focaliser l'attention uniquement sur les interactions entre jeunes, sans que ne soit réellement questionné ce qui se transmet effectivement dans la famille.

Les usages se déroulent cependant en grande partie au domicile parental. L'équipement familial, les usages, les temps d'accès doivent être négociés avec les parents et l'usage reste le plus souvent soumis à des formes de surveillance et de contrôle parentaux. Même si les adolescents apprennent surtout par mimétisme, avec leurs pairs, les usages des parents, leurs inquiétudes ou le rôle qu'ils accordent à l'informatique dans la réussite scolaire de leurs enfants ne sont pas sans incidence sur le rapport des jeunes à l'informatique et sur le processus d'appropriation. Ainsi, l'appropriation n'est pas seulement générationnelle, mais elle se déroule dans des contextes sociaux variés - amical certes, mais aussi familial ou scolaire. Cette appropriation plurielle fait supposer l'existence d'une pluralité dispositionnelle, en référence aux analyses de Bernard Lahire ${ }^{5}$. Mais existe-t-il pour autant des phénomènes de reproduction entre les générations similaires à ceux mis au jour concernant les pratiques culturelles ? Est-ce que " quelque chose " se transmet dans la famille concernant les usages des ordinateurs et, si oui, comment qualifier ce "quelque chose " ? Le point de vue adopté ici consiste à interroger les mécanismes de transmission familiale à la lumière du concept de capital culturel développé par Pierre Bourdieu ${ }^{6}$ et à questionner sa validité à pro-

${ }^{1}$ Frydel, 2006 ; Bigot, 2006.

${ }^{2}$ Le logiciel de messagerie instantané le plus utilisé par les jeunes adolescents.

${ }^{3}$ Metton, 2006 ; Pasquier, 2005.

${ }^{4}$ Singly (de), 2006.

${ }^{5}$ Lahire, 1998.

${ }^{6}$ Bourdieu, « Les trois états du capital culturel ", 1979. II a développé le concept de capital culturel afin de s'opposer aux explications de sens commun, renvoyant à un déterminisme biologique ou économique, pour expliquer l'élimination différentielle des élèves du système scolaire. 
pos de la transmission des gestes techniques et des dispositions relatifs à l'usage des ordinateurs.

Cet article est issu d'une recherche ethnographique ${ }^{7}$ menée pendant deux années dans un collège de banlieue parisienne ${ }^{8}$, qui vise à saisir les modalités d'appropriation des ordinateurs par les adolescents, par transmission familiale et scolaire et par circulation au sein des réseaux de pairs, au-delà de l'hypothèse d'un " bain " dans lequel seraient plongés les adolescents.

Nous montrerons que, pour ces jeunes adolescents, le processus même d'appropriation dans le contexte familial passe par l'établissement de tout un système de relations intrafamiliales reflétant l'autonomisation des usages de l'ordinateur, dont les ajustements renvoient à la distribution des compétences techniques et à leur manifestation sur la scène familiale.

\section{LA TRANSMISSION D'UN CAPITAL INFORMATIQUE}

Si l'on considère les ordinateurs dans leur dimension d'objets culturels, il n'est guère aisé de déterminer ce qui peut se transmettre entre les générations concernant leur usage. Ne dit-on pas les enfants plus compétents que leurs parents et que ce sont eux qui sont en mesure de leur " transmettre " des compétences techniques, au point que I'on parle de "rétrosocialisation ", la transmission $s^{\prime}$ effectuant des enfants vers les parents ${ }^{9}$ ? C'est certes le cas de certains parents - Dgibril (classe de sixième, milieu populaire) dit ainsi que sa mère voudrait bien qu'il " lui apprenne " - mais d'autres occupent en revanche la position d'expert familial. Les usages, représentations ou discours des parents sur les technologies varient également considérablement, selon leurs référents culturels, leurs usages personnels ou professionnels. Comment rendre compte de ces variations ? Pour cela, nous introduisons la notion de capital informatique, en supposant que, à I'instar du capital culturel, il peut être réinvesti sur différents marchés, scolaires, professionnels, etc., et qu'il existe sous trois formes essentielles

- matérielle ou objectivée (qui s'exprime par la possibilité d'accéder à des ressources informatiques matérielles, ordinateurs, réseau haut débit, etc.) ;

- incorporée (sous forme de savoir-faire pratiques, habiletés techniques, schèmes d'utilisation, dispositions, savoir-être social, compétences interactionnelles, etc.) ; - institutionnalisée (à travers la reconnaissance sociale des compétences informatiques par l'attribution de titres scolaires ou de diplômes ${ }^{10}$ ).

\footnotetext{
${ }^{7}$ Dans le cadre d'une thèse en sciences de l'éducation (École normale supérieure de Cachan/France Télécom R\&D). Les analyses présentées ici s'appuient sur des observations directes de situations d'usage et de sociabilité (dans la cour de récréation, en classe et dans un centre social attenant, les notes de terrain intégrées au corpus couvrant 50 séances, réalisées d'octobre 2005 à juin 2006), des conversations ethnographiques et des entretiens enregistrés. Parmi les collégiens étudiés, 43 ont été retenus dans le corpus final de l'enquête, dont 27 interrogés en situation d'entretien enregistré, au collège, de janvier 2006 à mai 2007 ; on compte 25 garçons pour 18 filles, 15 jeunes d'un milieu favorisé et 28 d'un milieu populaire. D'autres données recueillies ne sont pas exploitées ici.

${ }^{8}$ Plus de la moitié des élèves est issue de milieux défavorisés, moins d'un tiers de milieux favorisés (source : académie).

${ }^{9}$ Pasquier, 2005, p. 33.

${ }^{10}$ Du fait de la faible reconnaissance scolaire des compétences informatiques et du peu de valorisation des brevets scolaires comme le B2i (brevet informatique et Internet), cette forme institutionnalisée est particulièrement peu développée chez les jeunes, la reconnaissance sociale étant avant tout celle du groupe de pairs. Nous ne discuterons donc pas ici de cette forme, qui ne relève pas que de la famille.
} 
Nous faisons I'hypothèse que l'introduction de la notion de capital informatique permet de rendre compte de l'accès inégal aux différentes ressources permettant l'usage des outils informatiques et du maintien de disparités dans les accès, les usages et les compétences techniques ${ }^{11}$. Toutefois, le recours au modèle de la transmission ne signifie pas que les pratiques informatiques ou que les compétences se transmettraient d'une génération à l'autre à l'identique. Cela n'aurait aucun sens tant les usages des adolescents leur sont particuliers. Ce modèle ne renvoie pas non plus à une conception dans laquelle il y aurait d'un côté un émetteur (les parents) et de l'autre un récepteur passif (les enfants). Ces derniers jouent bien entendu un rôle actif et constructif, non seulement en réinventant de nouvelles formes culturelles, comme dans les blogs ${ }^{12}$, mais aussi en arbitrant ce qui "passe " ou non effectivement entre les générations. La transmission ne peut se jouer que dans un espace défini par l'établissement de la ligne de front de l'autonomie accordée

La prédominance de la socialisation horizontale dans le processus d'appropriation risque de focaliser l'attention uniquement sur les interactions entre jeunes, sans que ne soit réellement questionné ce qui se transmet effectivement dans la famille. aux adolescents, résultant de négociations parfois ardues. La " technicité " même des pratiques, les compétences, schèmes et savoir-faire, leur répartition au sein du foyer interviennent dans l'établissement et la reconfiguration permanente des relations familiales. Quelles sont donc les modalités du travail appropriatif qui s'y déroule?

Le fait que les parents soient ou non utilisateurs modifie considérablement les modalités de cette transmission familiale, qui diffère donc fortement selon les familles. Voir leurs parents utiliser un ordinateur, éprouver eux-mêmes des difficultés ou au contraire faire preuve d'une expertise technique, s'en servir pour communiquer ou pour travailler jouent un rôle important dans le rapport aux outils informatiques que développent les enfants.

\section{NÉGOCIER L'ÉQUUPEMENT DE LA FAMILLE}

Ce que les parents "transmettent " tout d'abord à leurs enfants est la possibilité d'utiliser du matériel informatique, d'accéder à Internet. En l'absence d'un ordinateur connecté à domicile, les adolescents doivent recourir à des stratégies pour accéder à leurs comptes MSN ou à leurs blogs, en se rendant chez des amis ou dans un cybercafé. Le fait que la grande majorité des foyers où résident des adolescents est désormais équipée ne saurait suffire à annuler la variabilité de cette forme objectivée du capital informatique.

La distinction binaire entre foyers équipés et foyers non équipés ne suffit pas à rendre compte de la diversité des configurations rencontrées. Tout d'abord, la généralisation actuelle des équipements tend à masquer les effets de l'ancienneté des usages à domicile, car si presque tous les jeunes rencontrés, quel que soit leur

\footnotetext{
${ }^{11}$ Lelong, Thomas, Ziemlicki, 2004 ; Alibert, Bigot, Foucaud, 2005.

${ }^{12}$ Fluckiger, 2006.
} 
milieu social, disposent désormais d'un ordinateur, ceux issus d'un milieu populaire sont souvent équipés depuis moins longtemps, parfois moins d'un an. Ils se souviennent de la date de l'achat, de l'impatience d'acquérir un ordinateur ou une connexion à Internet: "En fait je voulais Internet, j'ai attendu longtemps avant d'avoir Internet. " (Ilham, classe de quatrième, milieu populaire.) Cette situation contraste avec celle d'adolescents d'origine favorisée, dont certains disposent d'un ordinateur au foyer depuis leur enfance et ont commencé à jouer à des jeux éducatifs avant de passer aux usages adolescents : "Quand j'étais petit je faisais des coloriages sur l'ordinateur aussi. [...] Maintenant je fais plus du tout ça hein [rires]. " (Clément, classe de sixième, milieu favorisé.) II s'amusait également à " taper une histoire " dans Word: "J'avais tapé sept pages d'ordinateur, pour moi c'était immense. Je tapais à peu près dix lignes à chaque fois que j'en faisais, mais j'en faisais pas très souvent. " La " carrière informatique " de ces jeunes issus de milieux déjà utilisateurs commence donc beaucoup plus tôt que celle d'adolescents dont la famille est démunie en capital informatique. Chez eux, le capital informatique est ancré plus précocement, ce qui facilite la prise de conscience du caractère transitoire, lié aux débuts de l'adolescence, de leurs propres usages : ces adolescents savent qu'il existe d'autres formes d'usages que ceux partagés par les jeunes. Les jeunes issus d'une famille populaire non utilisatrice, souvent équipée récemment, étant les porteurs familiaux des usages face à des parents qui se disent dépassés, doivent en revanche inventer leurs propres usages, leur propre rapport à la technologie informatique, la place particulière que celle-ci prend dans l'accès aux produits culturels et dans la sociabilité.

La localisation des équipements a également son importance et posséder un ordinateur personnel est une revendication courante et le signe de l'autonomie accordée par les parents : " II est dans la chambre des parents, c'est pour ça que je veux un ordinateur portable. " (Gaëlle, classe de cinquième, milieu favorisé.) Aussi, les familles déjà équipées en ordinateurs (c'est le cas le plus fréquent dans les milieux favorisés) peuvent choisir de dupliquer les équipements informatiques ${ }^{13}$ pour que les enfants aient la possibilité d'accéder plus facilement aux ressources numériques. Lors de l'achat d'un nouvel ordinateur, I'ancien a été donné à Justine (classe de cinquième, milieu favorisé) et installé dans sa chambre, mais seul le nouveau étant connecté à Internet, les usages possibles de l'ancien sont limités: "En fait sur le mien je l'utilise pas trop, parce qu'il y a pas Internet. Sur le nouveau, je vais sur MSN. " La duplication des ordinateurs suit davantage les étapes du renouvellement du matériel informatique qu'une réelle organisation réfléchie de la répartition du matériel entre les enfants. Clément raconte les différentes étapes de l'équipement du foyer: "Au début l'ordinateur était dans la chambre de mon frère, on avait un seul ordinateur, c'était un pour deux, mais, quand même, on avait un ordinateur. Et après, quand ça se modernise, on change. Après moi j'en ai eu un, mon frère il a gardé le vieux, après c'est le vieux qui a été jeté et puis j'en ai encore eu un nouveau parce que c'est mon grand-père qui s'en est racheté un nouveau aussi, donc il m'a donné l'ancien, qui est pas mal quand même et puis mon frère, bientôt, mon père, il va lui donner son ancien ordinateur portable. En même temps il en a un très vieux [rires], très, très vieux. "

\footnotetext{
${ }^{13}$ Pharabod, 2004 ; Le Douarin, 2007.
} 
L'équipement des enfants semble ici obéir à une règle d'alternance destinée à respecter l'équité entre les enfants. Les parents sont également priés d'acheter un nouvel ordinateur quand l'ancien ne permet plus de jouer aux mêmes jeux que les copains, ou de se connecter à Internet. Si les parents de Lucie (classe de troisième, milieu populaire) ont acquis de nouveaux ordinateurs, c'est parce que : " [...] en fait, on joue à un jeu et y avait le dernier modèle, il marchait pas sur l'ancien, donc ils ont acheté celui-là et en même temps, mon frère, il s'en est acheté un. "

L'inégale répartition du capital objectivé tient enfin à la place physique et symbolique des équipements informatiques au sein du foyer. Comme dans le cas de la télévision ${ }^{14}$, les configurations spatiales rencontrées renvoient à la place symbolique que l'on accorde à ces équipements. Mais la place objective dans les pratiques culturelles ou de communication des adolescents varie également avec la localisation des équipements et l'intimité plus ou moins grande qu'elle offre. Dans les familles populaires, I'ordinateur est plus souvent situé dans un espace collectif, salon ou entrée, et les acti-

Les jeunes issus d'un milieu favorisé, dont les parents sont souvent eux-mêmes utilisateurs, ont conscience que leurs usages sont liés à leur âge et c'est par la spécificité des usages juvéniles qu'ils se distinguent des adultes et marquent leur statut d'adolescent.

vités informatiques se déroulent donc sous le regard des parents. Or, tenir un blog ou converser sur MSN sont tenus par les adolescents pour une activité " privée ", au sens où Oliver Schwartz ${ }^{15}$ entend l'intime, le caché, ce qui est soustrait au regard des autres. II s'agit donc pour les adolescents de conquérir des usages autonomes.

\section{LA CONQUETE DE SON AUTONOMIE}

Les formes de contrôle parental, la négociation de la privatisation des usages et d'un territoire personnel délimitent la transmission du capital incorporé sur deux plans : d'abord en définissant un espace de liberté, une "zone libre ${ }^{16}$ " dans laquelle les usages peuvent s'épanouir, ensuite parce qu'à travers les formes de contrôle, c'est un certain rapport aux technologies informatiques que les parents tentent d'inculquer à leurs enfants, qui joue comme facteur différenciant dans les dispositions acquises.

Comme le rappelle François de Singly, " les adonaissants ne sont que partiellement propriétaires d'eux-mêmes, même pour les activités qu'ils contrôlent plus directement ${ }^{17}$ ". Même pour des usages informatiques que les jeunes peuvent considérer comme étant leur domaine réservé, le pouvoir de décision parental reste fort. Ces inquiétudes portent sur plusieurs genres de risques, comme l'accès à des contenus inadaptés, notamment pornographiques, les virus ou la publication de photographies ou d'informations personnelles sur le Web ${ }^{18}$.

\footnotetext{
${ }^{14}$ Schwartz, 1989. II a notamment montré l'importance de la place symbolique et physique occupée par la télévision dans les milieux ouvriers.

${ }^{15}$ Schwartz, 1989.

${ }^{16}$ Pour reprendre l'expression de François de Singly (2006).

${ }^{17}$ Singly (de), 2006.

${ }^{18}$ Metton, 2006.
} 
Le premier élément que les parents peuvent contrôler est l'accès physique aux équipements informatiques. Même lorsque l'ordinateur est acheté et installé au domicile, les parents continuent d'exercer un contrôle sur l'accès des enfants à I'ordinateur. Beaucoup de parents estiment que leurs enfants passent trop de temps sur MSN et Internet et cherchent à en limiter les usages. La mère de Christian (classe de troisième, milieu populaire) débranche l'ordinateur : "Des fois ce qu'elle fait, c'est qu'elle débranche tout sur l'ordinateur, elle prend la souris [...], elle éteint, tu vas pas sur l'ordinateur, pendant trois jours, deux jours même, je peux pas. "Celle d'Iris (classe de troisième, milieu favorisé) confisque le modem, qu'elle conserve dans un placard fermé à clé. Une part importante de la négociation de l'accès physique aux équipements consiste ainsi en un combat quotidien pour un usage ludique et communicationnel, face à des parents qui tentent d'imposer des règles et de marquer des limites à l'autonomie accordée aux adolescents.

Le problème de la conquête d'un accès au capital objectivé se pose très différemment selon que les parents sont utilisateurs ou non et selon le caractère plus ou moins permissif de leur politique éducative. Les conditions concrètes du contrôle sont très dépendantes de la distribution de l'expertise technique au sein du foyer et des jeux relationnels intrafamiliaux qui y prennent appui. Des parents non utilisateurs peuvent par exemple ne pas avoir techniquement les moyens de contrôler les usages de l'ordinateur de leurs enfants mais se montrer plus restrictifs sur les temporalités des usages. Certains parents font le choix d'interdire certaines pratiques, notamment $\mathrm{MSN}$, qui supporte une partie importante des craintes parentales; plusieurs adolescents font état de son interdiction à la maison. Cette volonté de surveillance est clivée, à la fois socialement et par le genre. Certaines filles de milieux populaires font état d'un contrôle spécifique. La mère de Samia (classe de troisième, milieu populaire) surveille ses discussions sur MSN : "Elle veut bien voir si j'ai des contacts avec des garçons, des choses comme ça [...], elle regarde le plus souvent les conversations enregistrées. " Pour cela, elle consulte I'historique des conversations de sa fille dans le logiciel MSN. Samia a dû modifier son mot de passe : "Des fois je mets des codes, des choses comme ça $[. .$.$] , comme un jour elle connaissait mon code, après je l'ai changé,$ comme ça après elle pouvait plus rien voir. "Si sa mère connaissait le mot de passe, c'est parce que : "Au départ, c'est elle qui faisait mon adresse et tout, et je lui disais ce qu'elle devait écrire, après elle faisait, après quand elle faisait mon adresse, je lui disais mon code. Maintenant je l'ai changé, maintenant je lui dis plus, elle s'énerve, mais moi je vais pas lui donner mon code. Je sais pas, mais moi j'ai envie d'intimité aussi, pourquoi elle vient toujours comme ça ? " Ainsi, la connaissance des mots de passe était due à la dépendance de Samia, qui ne savait pas créer et gérer elle-même un compte MSN. Avec l'adolescence et le besoin d'une intimité plus importante, Samia a dû acquérir de nouvelles compétences afin de ne plus dépendre de sa mère et de privatiser une partie de ses usages en les soustrayant au regard parental. C'est ici dans l'accroissement des compétences techniques que s'ancre ce processus d'autonomisation et d'émancipation en cours à l'adolescence.

C'est Christian en revanche qui gère I'ordinateur familial, que ses parents utilisent peu et dont il est I'utilisateur principal. Cette position rend très difficile le 
contrôle a posteriori de ses activités informatiques : "Quand je suis là, elle [sa mère] vient, elle guette, mais quand je suis pas là, elle sait même pas comment s'en servir. "La seule chose sur laquelle sa mère peut jouer est le temps d'usage : il n'utilise l'ordinateur qu'en son absence et l'éteint quelques minutes avant son retour du travail, à $21 \mathrm{~h} 30$.

\section{LA VARIABILITÉ DU CAPITAL INCORPORÉ}

Les formes de la transmission du capital informatique diffèrent également fortement selon les familles. La nature de l'aide apportée aux jeunes adolescents varie selon qu'elle est le fait de voisins sollicités à l'occasion d'un problème ponctuel ou de parents qui cherchent à établir une relation pédagogique. Yacine (classe de cinquième, milieu populaire) raconte : "À un moment, mon ordinateur, quand on l'allumait, il s'allumait pas très bien et comme mon voisin c'était un ingénieur en informatique, ben il est venu et il nous a tout ramené bien. "En

La famille ne peut être envisagée uniquement comme instance d'inculcation, mais comme lieu principal de l'individualisation du rapport aux ordinateurs.

revanche des parents utilisateurs chevronnés peuvent instaurer une démarche explicite d'apprentissage. Le père de Clément, enseignant à l'université, lui a montré le fonctionnement d'une base de données qu'il utilise dans son travail : "II m'avait montré, j'en avais fait une petite, moi. " Clément n'en a jamais eu l'usage, mais connaissant son existence, il peut prendre conscience du monde pour I'instant inexploré que recèle l'ordinateur. Ces moments sont souvent l'occasion pour ces adolescents d'entendre un vocabulaire technique qu'ils ont moins l'occasion d'apprendre avec leurs amis. Clément connaît ainsi le nom de certains logiciels extérieurs à la culture informatique juvénile : "Mon père, des fois, il a installé des logiciels en cas si l'ordi, il tombe en panne, il pourra travailler sur le mien... il s'est installé des logiciels pour lui [...]. II y a... Python... [...] C'est un truc... bizarre... j'ai jamais utilisé... ou Adobe FriendMaker, il a installé ça. Ou sinon il y a PowerPoint. " Son attitude contraste avec celle d'autres jeunes qui, en cas de panne, se contentent de redémarrer l'ordinateur avant de renoncer, comme Kader (classe de sixième, milieu populaire) : " II bug là... ouais, mais là il marche pas. [Comment ça se fait ?] Je sais pas, c'est mal fait, c'est ma sœur, elle a fait je sais pas quoi et il marche pas. [...] Elle me dit quoi, que ça marche pas, après je lui dis, je sais pas le régler. "À l'inverse, quand Clément rencontre un problème: " Je le dis à mes parents, mais j'essaie de le résoudre avant, tout seul... j'essaie, oui hein [...], je regarde qu'est-ce qui marche, j'essaie de savoir où est le problème, et sinon j'appelle mon père quand je trouve pas, puis ma mère aussi. " Le capital incorporé de Clément consiste donc non seulement en une connaissance plus approfondie des logiciels et procédures informatiques, mais aussi en une disposition à dépasser le simple engagement pragmatique qui caractérise les usages de presque tous les collégiens rencontrés et à considérer l'ordinateur dans sa dimension d'objet technique.

Les jeunes de milieux favorisés peuvent également découvrir chez eux, parallèlement aux usages générationnels, des usages plus proches de ceux valorisés par l'institution scolaire que les chats, blogs, jeux et sites Web ludiques qui 
constituent l'essentiel des usages juvéniles. Les parents de Clément " utilisent tout le temps Word, au travail, même à la maison ". La mère de Lisa (classe de troisième, milieu favorisé) lui a appris les fonctionnalités de base de l'ordinateur ou I'usage de Word et du tableur-grapheur : "Elle m'a appris à utiliser les tableursgrapheurs, etc. [...] C'est basique ce que je fais hein... en général, c'est simple. Ma mère m'a montré comment faire des jolis titres, alors depuis, je fais comme ça et puis ça marche très bien. Enfin pour le rapport de stage, c'était galère, parce que comme il y avait trente pages, pour gérer les trente pages en même temps... c'est compliqué. Mais bon, ma mère m'a aidée pour la mise en pages parce qu'il y avait des pages, des fois, elles disparaissaient, elles réapparaissaient pas au bon endroit. "

Les usages ne semblent en revanche pas s'inscrire dans des stratégies de " distinction ${ }^{19}$ " sociale : I'émancipation des valeurs familiales passe en premier lieu par l'adhésion au groupe et uniformise les usages. Les jeunes adolescents cherchent à rendre visible l'évolution de leur " taille symbolique ${ }^{20}$ " et I'usage même des outils de communication ou des blogs est perçu comme un signe distinctif de leur génération, un attribut qui atteste de leur statut de " jeune ». Si cette stratégie de distinction générationnelle existe pour la plupart des jeunes, elle semble cependant opérer différemment selon les milieux sociaux. Les jeunes issus d'un milieu populaire sont souvent les principaux utilisateurs des ordinateurs familiaux, parfois également reconnus comme " experts" au sein de la famille. Pour eux, le simple fait d'utiliser l'ordinateur est un signe distinctif du statut d'adolescent. Comme les adultes de leur entourage utilisent moins les ordinateurs qu'eux-mêmes, les usages ludiques ou communicationnels des ordinateurs sont pour eux les seuls usages connus. À l'inverse, les jeunes issus d'un milieu favorisé, dont les parents sont souvent eux-mêmes utilisateurs, ont conscience que leurs usages sont liés à leur âge et c'est par la spécificité des usages juvéniles qu'ils se distinguent des adultes et marquent leur statut d'adolescent.

\section{CONCLUSION}

La transmission familiale d'un capital informatique - ou culturel - dépend de conditions objectives, notamment de l'existence d'un temps de socialisation commun aux parents et aux enfants ${ }^{21}$. La spécificité des usages des adolescents, qui y cherchent un moyen d'émancipation de la tutelle familiale et de manifestation de leur statut, ne facilite bien évidemment pas l'existence de ce temps. Un adolescent utilisant MSN pour communiquer avec ses amis marque justement qu'il est désormais un " grand " en prolongeant les relations de pairs au sein même du domicile familial22. II supporte de ce fait difficilement la surveillance parentale et ses pratiques de communication, si elles sont " publiques " pour son réseau amical, restent relativement " privées " pour ses parents.

\footnotetext{
${ }^{19}$ Bourdieu, La distinction, 1979.

${ }^{20}$ Singly (de), 2006.

${ }^{21}$ Lahire, 1995.

${ }^{22}$ Metton, 2006.
} 
De ce fait, les occasions d'une socialisation familiale sont rares. Beaucoup plus que pour la télévision, qui offre de multiples occasions de pratiques collectives et qui permet de construire une culture familiale commune ${ }^{23}$, les pratiques informatiques sont individuelles. S'il existe de véritables utilisations collectives des ordinateurs entre pairs visitant ensemble les mêmes sites, s'inscrivant ensemble sur un chat, visitant les Skyblogs ou jouant à un jeu, les exemples d'activités conjointes intergénérationnelles sur l'ordinateur sont en revanche très rares. II s'agit presque toujours de parents utilisateurs chevronnés, qui cherchent à instaurer une relation pédagogique par une démarche explicite d'apprentissage. Mais passé le moment de l'apprentissage d'un savoir-faire particulier, chacun reprendra ses activités habituelles sur l'ordinateur familial : les parents consulteront leur courrier électronique, effectueront des démarches administratives ou liront la presse, les enfants consulteront leur liste de contact MSN et les nouveaux commentaires sur leur blog.

Ainsi, au-delà de la simple possibilité d'accéder aux ressources matérielles, la transmission d'un capital informatique incorporé se heurte à des difficultés pratiques et les usages juvéniles apparaissent davantage marqués par l'adolescence que par l'appartenance à différents milieux sociaux. Cependant, à travers des ajustements parfois problématiques et une coordination des conduites nécessitant de constantes renégociations, "quelque chose " passe effectivement entre les générations, concernant le rapport aux ordinateurs. Ce ne sont à proprement parler ni des compétences techniques ni des pratiques qui se transmettent, mais bien davantage des dispositions ou des inclinaisons, qui se construisent dans un espace de liberté défini dans la négociation de la privatisation des usages. La famille ne peut être envisagée uniquement comme instance d'inculcation, mais comme lieu principal de l'individualisation du rapport aux ordinateurs. Et si cette individualisation n'est pas indépendante de la transmission d'un capital spécifique à l'usage des outils informatiques, elle ne peut bien entendu s'y réduire : le rapport que construisent les adolescents au sein de leur famille dépend également de leur trajectoire d'usage idiosyncrasique et des contingences liées à leur position personnelle en tant qu'adolescents au sein du groupe de pairs.

\section{Bibliographie}

Alibert D., Bigot R., Foucaud D., La dynamique des inégalités en matière de nouvelles technologies : méthodes d'approche, analyse évolutive, Centre de recherche pour l'observation et l'étude des conditions de vie, Paris, 2005.

Bıgot R, La diffusion des technologies de l'information dans la société française, Centre de recherche pour l'étude et l'observation des conditions de vie, Paris, 2006 (téléchargeable à l'adresse : www.arcep.fr/uploads/tx_gspublication/etude-credoc2006.pdf).

Bourdieu P., La distinction, Minuit, Paris, 1979.

BOURDIEU P., "Les trois états du capital culturel ", Actes de la recherche en sciences sociales, $\mathrm{n}^{\circ}$ 30, 1979, pp. 3-6.

FLUCKIGER C., "La sociabilité juvénile instrumentée : l'appropriation des blogs dans un groupe de collégiens ", Réseaux, n¹38, vol. XXIV, 2006, pp. 111-138.

${ }^{23}$ Proulx, Laberge, 1995. 
FRYDEL Y., «Internet au quotidien : un Français sur quatre ", Insee première, n 1076, 2006.

LAHIRE B., Tableaux de famille, Le Seuil/Gallimard, Paris,1995.

LAHIRE B., L'homme pluriel : les ressorts de l'action, Nathan, Paris, 1998.

Le Douarin L., Le couple, l'ordinateur, la famille, Payot, Paris, 2007.

Lelong B., Thomas F., ZIEMLICKI C., “ Des technologies inégalitaires ? L'intégration de I'Internet dans l'univers domestique et les pratiques relationnelles ", Réseaux, n ${ }^{\text {os }}$ 127-128, vol. XXII, 2004, pp. 141-180.

METTON C., Devenir grand : le rôle des technologies de la communication dans la socialisation des collégiens, thèse de sociologie, École des hautes études en sciences sociales, 2006.

PAsquier D., Cultures lycéennes : la tyrannie de la majorité, Autrement, Paris, 2005.

Pharabod A.-S., “Territoires et seuils de l'intimité familiale ", Réseaux, n 123, vol. XXII, 2004, pp. 85-111.

Proulx S., LABerge M.-F., «Vie quotidienne, culture télévisuelle et construction de l'identité familiale ", Réseaux, n 70, 1995.

SchWARTZ O., Le monde privé des ouvriers, Presses universitaires de France, Paris, 1989.

Singly F. (de), Les adonaissants, Armand Colin, Paris, 2006. 